\title{
Quantitative changes in perineuronal nets in development and posttraumatic condition
}

\author{
Nikita Lipachev ${ }^{1,2} \cdot$ Nikita Arnst $^{1,3} \cdot$ Anastasiia Melnikova ${ }^{1,4} \cdot$ Harri Jäälinoja $^{5}$ Anastasiya Kochneva ${ }^{4}$. \\ Alexander Zhigalov ${ }^{1} \cdot$ Natalia Kulesskaya ${ }^{1} \cdot$ Albert V. Aganov $^{2} \cdot$ Mikhail Mavlikeev $^{4} \cdot$ Heikki Rauvala $^{1}$. \\ Andrey P. Kiyasov ${ }^{4} \cdot$ Mikhail Paveliev $^{1,6}$
}

Received: 28 November 2018 / Accepted: 11 March 2019 / Published online: 22 March 2019

(c) The Author(s) 2019

\begin{abstract}
Perineuronal net (PNN) is a highly structured portion of the CNS extracellular matrix (ECM) regulating synaptic plasticity and a range of pathologic conditions including posttraumatic regeneration and epilepsy. Here we studied Wisteria floribunda agglutinin-stained histological sections to quantify the PNN size and enrichment of chondroitin sulfates in mouse brain and spinal cord. Somatosensory cortex sections were examined during the period of PNN establishment at postnatal days 14, 21 and 28. The single cell PNN size and the chondroitin sulfate intensity were quantified for all cortex layers and specifically for the cortical layer IV which has the highest density of PNN-positive neurons. We demonstrate that the chondroitin sulfate proteoglycan staining intensity is increased between P14 and P28 while the PNN size remains unchanged. We then addressed posttraumatic changes of the PNN expression in laminae 6 and 7 of cervical spinal cord following hemisection injury. We demonstrate increase of the chondroitin sulfate content at 1.6-1.8 $\mathrm{mm}$ rostrally from the injury site and increase of the density of PNN-bearing cells at 0.4-1.2 mm caudally from the injury site. We further demonstrate decrease of the single cell PNN area at $0.2 \mathrm{~mm}$ caudally from the injury site suggesting that the PNN ECM takes part in the posttraumatic tissue rearrangement in the spinal cord. Our results demonstrate new insights on the PNN structure dynamics in the developing and posttraumatic CNS.
\end{abstract}

Keywords Perineuronal nets $\cdot$ Extracellular matrix $\cdot$ Somatosensory cortex $\cdot$ Brain development $\cdot$ Spinal cord injury

Electronic supplementary material The online version of this article (https://doi.org/10.1007/s10735-019-09818-y) contains supplementary material, which is available to authorized users.

Mikhail Paveliev

paveliev@outlook.com

1 Neuroscience Center, University of Helsinki, Viikinkaari 4, P.O.Box 56, 00790 Helsinki, Finland

2 Institute of Physics, Kazan Federal University, Kazan Kremlyovskaya 16a, Tatarstan, Russia 420111

3 Institut für Biochemie und Biophysik, Friedrich-Schiller-Universität Jena, Hans-Knöll-Str.2, 07745 Jena, Germany

4 Institute of Fundamental Medicine and Biology, Kazan Federal University, Kazan K.Marx 74, Tatarstan, Russia 420012

5 Institute of Biotechnology, University of Helsinki, Viikinkaari 9, P.O.Box 56, 00790 Helsinki, Finland

6 Danish Research Institute of Translational Neuroscience, Aarhus University, Ole Worms Allé 3, 8000 Aarhus C, Denmark

\section{Introduction}

Perineuronal net is a highly condensed portion of the brain extracellular matrix (ECM) surrounding cell somata and proximal processes of substantial neuronal populations in various regions of the brain and spinal cord (Kwok et al. 2011; Shen 2018). Over the last decades perineuronal nets attracted remarkable attention of neuroscientists for their crucial role in the regulation of the CNS plasticity during postnatal development and posttraumatic regeneration (Alilain et al. 2011; Massey et al. 2006; Kwok et al. 2011). PNN is a crucial functional component of the CNS synapses (Korotchenko et al. 2014; Dityatev and Rusakov 2011) that undergoes major changes during early postnatal development and the brain synaptic circuitry maturation (Carulli et al. 2016). PNN formation is induced by synaptic inputs to the developing brain neuronal network (Lander et al. 1998; Brückner et al. 2004; McRae et al. 2007; Carulli et al. 2010). After the PNN is formed it in turn starts to control synaptic 
plasticity (Pizzorusso et al. 2002; Corvetti and Rossi 2005; Miyata and Kitagawa 2015).

Wisteria floribunda agglutinin (WFA) is commonly used to detect the $N$-acetyl-galactosamine (GalNAc) residues within chondroitin sulfate chains (Härtig et al. 1992). WFA staining is abrogated by the chondroitinase ABC (ChaseABC) treatment (Fawcett 2015) or by the ablation of the major PNN chondroitin sulfate proteoglycan (CSPG) aggrecan (Kwok et al. 2010; Giamanco et al. 2010). In a number of studies using ChaseABC CSPGs were demonstrated to be the central molecular component of PNN restricting synaptic plasticity during the closure of the critical period and in the adult CNS (Fawcett 2015; García-Alías et al. 2009; Pizzorusso et al. 2002). At the same time quantitative comparison of the chondroitin sulfate enrichment in the PNN at different developmental stages remains largely unaddressed.

Acute injury to the brain or spinal cord causes a complex cascade of changes in cell biochemistry, morphology, cell-cell interaction and tissue architecture (Burda et al. 2016). Neuronal networks undergo profound plasticity including compensatory sprouting and regeneration attempts (Cregg et al. 2014). The role of PNN in the regulation of posttraumatic regeneration has been studied in the model of spinal cord dorsal column transection followed by the brainstem nuclei deafferentation (Massey et al. 2006), spinal cord hemisection (Alilain et al. 2011) and peripheral nerve injury (Galtrey et al. 2007).

While the physiological importance and protein content of PNN received much attention over the last decade (Kwok et al. 2011; Miyata and Kitagawa 2015), quantitative studies on the PNN microstructure and chondroitin sulfate enrichment in development and posttraumatic condition are largely missing. Here we used quantitative image analysis to reveal changes of the PNN area and the CSPG enrichment in the developing somatosensory cortex and the posttraumatic spinal cord.

\section{Materials and methods}

\section{Brain tissue preparation}

Mouse brain samples of postnatal day 14, 21 and 28 were collected according to regulations of the ethics committee of Kazan Federal University. All applicable institutional guidelines for the care and use of animals were followed. All procedures performed in studies involving animals were in accordance with the ethical standards of the institution at which the studies were conducted. For immunohistochemistry animals were terminally anaesthetized with an intraperitoneal overdose of Urethane (Sigma-Aldrich) and were immediately perfused through the heart with $30-50 \mathrm{ml}$ of ice-cold phosphate-buffered saline (PBS, $\mathrm{pH}$ 7.4), followed by $30-50 \mathrm{ml}$ of ice-cold $4 \%$ paraformaldehyde applied at $10 \mathrm{ml} / \mathrm{min}$.

For the cortex dissection mice were decapitated and the scull was cut from foramen magnum to lambda with microsurgery scissors. After that interparietal, parietal and frontal bone plates were carefully removed with microsurgery tweezers to expose the cortex. The dissection was controlled with a binocular microscope. In the case of tissue damage samples were excluded from the experiment.

Brains were postfixed overnight in $4 \%$ paraformaldehyde at $4{ }^{\circ} \mathrm{C}$. After that brains were cryoprotected with $30 \%$ sucrose for $48 \mathrm{~h}$ and then frozen in embedding Tissue-Tek medium (Sakura).

To control precise orientation of the Tissue-Tek-embedded samples we applied fine grids on the embedding forms (Sakura) to mark the border of the tissue sample location. Then a few drops of Tissue-Tek was applied to the form and cooled down till it became highly viscous. After that the brain or spinal cord sample was positioned inside the form according to the grid. High viscosity of the embedding medium made it possible to control the tissue orientation and prevent the spinal cord bending along the dorsoventral axis. Finally the sample was fully embedded in Tissue-Tek and incubated in a box with dry ice until complete freezing (about $30 \mathrm{~min}$ ). After that the forms with samples we transferred to $-80^{\circ} \mathrm{C}$. The samples were kept at $-80^{\circ} \mathrm{C}$ in a paper box for 1-2 days before cryosectioning.

$20 \mu \mathrm{m}$ thick coronal brain sections were cut with a Thermo Cryotome FE \& FSE A78910100 at the temperature -12 to $-15{ }^{\circ} \mathrm{C}$. The somatosensory cortex regions were determined using Comparative Cytoarchitectonic Atlas of Mouse Brain (Patrick R. Hof, Elsevier), bregma 0 to $-1 \mathrm{~mm}$. Sections were placed on Menzel-Gläser supefrost plus slides (Thermo Scientific) and air dried at room temperature for $10 \mathrm{~min}$ prior to WFA staining detailed below.

\section{Brain tissue staining}

The biotinylated Wisteria floribunda agglutinin (VectorLab, USA) was used for the PNN staining. After sectioning samples were washed three times using phosphate buffered saline (PBS, pH 7.4) to remove embedding medium. Then sections were treated for $1 \mathrm{~h}$ with the blocking solution: $5 \%$ bovine serum albumin (BSA, Sigma) in $0.1 \mathrm{M}$ PBS. To block endogenous biotin, a streptavidin/biotin Blocking Kit (Vector Lab, USA) was used according to the manufacturers protocol. After that the sections were quickly rinsed in PBS and incubated overnight at $+4{ }^{\circ} \mathrm{C}$ with the biotinylated WFA at a final concentration $2 \mu \mathrm{g} / \mathrm{ml}$ (dilution 1:1000) in PBS with $10 \mathrm{mM}$ HEPES, $\mathrm{pH}$ 7.4. Sections were washed 4-5 times for 5 min with PBS and incubated for $30 \mathrm{~min}$ with AlexaFluor 633-conjugated streptavidin (Invitrogen) (dilution 1:100) in PBS (pH 7.4). Finally the sections were 
washed 4-5 times for 5 min with PBS, air-dried and coverslipped with ImmunoMount (Thermo Scientific). To control for the staining specificity brain sections were treated with AlexaFluor 633-conjugated streptavidin, but not with WFA (Online Resource 1).

\section{Spinal cord injury}

All experimental protocols for the spinal cord injury experiments were approved by ELLA- Animal Experiment Board in Finland (the permission numbers: ESLH-2008-09065/ YM-23 and ESAVI/11326/04.10.07/2014). 1 month old $\mathrm{C} 57 \mathrm{bl} / 6$ female mice were anaesthetized with an intraperitoneal mixture of ketamine $(80 \mathrm{mg} / \mathrm{kg})$ and xylazine $(10 \mathrm{mg} /$ $\mathrm{kg})$. After laminectomy at C5 vertebral level, the dura was carefully removed. The right cervical spinal hemicord was transected with a $25 \mathrm{G}$ syringe needle from midline dorsal vessel to the lateral side. Control animals got a sham surgery that included laminectomy while the spinal cord and spinal cord's membrane left intact. After the suturing of skin and muscles mice were kept in warm recovery cage for 2-4 h until animals regain ability to move. Animals were treated with Rimadil ( $5 \mathrm{mg} / \mathrm{kg}$, Pfizer) and Rapidexon $(0.2 \mathrm{mg} / \mathrm{kg}$, Evrovet) to reduce pain and inflammatory swelling for the next 3-4 days after surgery until animals stopped to lose body weight. A few animals were in severe health condition as a result of the injury operation-those were not able to move within 3 days after spinal cord injury and lost more than $20 \%$ of body weight. Those animals were excluded from the analysis.

\section{Spinal cord tissue preparation}

At 9 weeks after injury animals were anaesthetized with carbon dioxide before surgical procedures. After that, as soon as mouse reflexes disappeared, mice were perfused through the heart with ice-cold PBS buffer ( $\mathrm{pH} 7.4 ; 50 \mathrm{ml}$ per mouse) and 4\% paraformaldehyde in PBS (pH 7.4, $25 \mathrm{ml}$ per mouse).

For the spinal cord preparation the cervical region of the vertebral column was dissected from the body. Dorsal portions of the neural arches were carefully removed with microsurgery tweezers under a binocular microscope. Intact dura mater and the absence of visible damage were used as the criteria of proper dissection.

Finally, the pieces of spinal cord were cryoprotected with $30 \%$ sucrose for $48 \mathrm{~h}$ at $+4{ }^{\circ} \mathrm{C}$ and then snap-frozen on dry ice in embedding Tissue-Tek medium. Then the $20 \mu \mathrm{m}$ thick longitudinal horizontal sections were cut with cryotome (Leica, Germany). Sections at the level of the central channel were selected from the entire set of sections.

\section{Spinal cord staining procedure}

Free-floating modification of the previously described PNN staining protocol (Arnst et al. 2016) was used in the present study. The biotinylated WFA (Vector Lab, USA) was used at a 1:500 dilution and the Alexa Fluor 633-conjugated streptavidin at a 1:100 dilution. Mouse monoclonal antibodies (Swant) were used for the parvalbumin (PV) staining of spinal cords. The secondary antibody was goat anti-mouse, Alexa Fluor 488-conjugated (Thermo-Fisher Scientific).

\section{Microscopy}

Epifluorescent images were collected for the PNN analysis. Brain sections were imaged with Zeiss Axio Imager 2.0 microscope with Plan-Apochromat x20/0.8 objective and Zeiss AxioCam HRc digital camera (Carl Zeiss Microscopy), pixel size $0.51 \mu \mathrm{m}$. In each experiment imaging settings were selected for P28 mouse brain samples so that the brightest PNN were not overexposed. The same settings were then used for P14 and P21 samples. Spinal cord sections were imaged with $\times 10 / 0.6$ objective, pixel size $0.645 \mu \mathrm{m}$. To control for the tissue section thickness confocal microscopy was performed with Zeiss LSM710 microscope, objective Plan-Apochromat 40x/1.4, voxel size $0.3 \times 0.3 \times 0.3 \mu \mathrm{m}$. Confocal stacks with $\mathrm{z}$ dimension of 30 $\mu \mathrm{m}$ were collected and 5 regions of interest, $200 \times 150 \times 30$ $\mu \mathrm{m}$ were selected in each stack for $3 \mathrm{D}$ reconstruction with Imaris software and measurements of the section thickness (Online Resource 2).

\section{Image analysis}

FIJI software (Schindelin et al. 2012) was used for image analysis. The PNN-labelled area and intensity parameters were used to quantify the enrichment of chondroitin sulfates. For the comparison of the PNN area and the intensity values at different distances from the spinal cord injury, the parameters $\left(I_{n}-I_{\text {mean }}\right) / I_{\text {mean }}$ and $\left(A_{n}-A_{\text {mean }}\right) / A_{\text {mean }}$ were quantified, where $I_{\text {mean }}$ is an average single cell PNN intensity value for each section, $A_{\text {mean }}$ is an average single cell PNN area value for each section, $I_{n}$ and $A_{n}$ are the PNN intensity and area values for individual cell. Statistical analysis is presented for at least 3 independent experiments using one-way ANOVA (Excel, Microsoft). In the spinal cord injury experiments paired $t$ test was used for pairwise comparison of data points at various distances from the injury site. We used a custom implementation of the permutation test (Welch 1990) to assess significance of the differences of the PNN area, intensity and PNN-positive cell density values at different distances from the spinal cord injury. Permutation tests were run with MatLab. Error bars represent standard error of mean (SEM), symbols *, ** and $* * *$ represent $P<0.05,0.01$ 

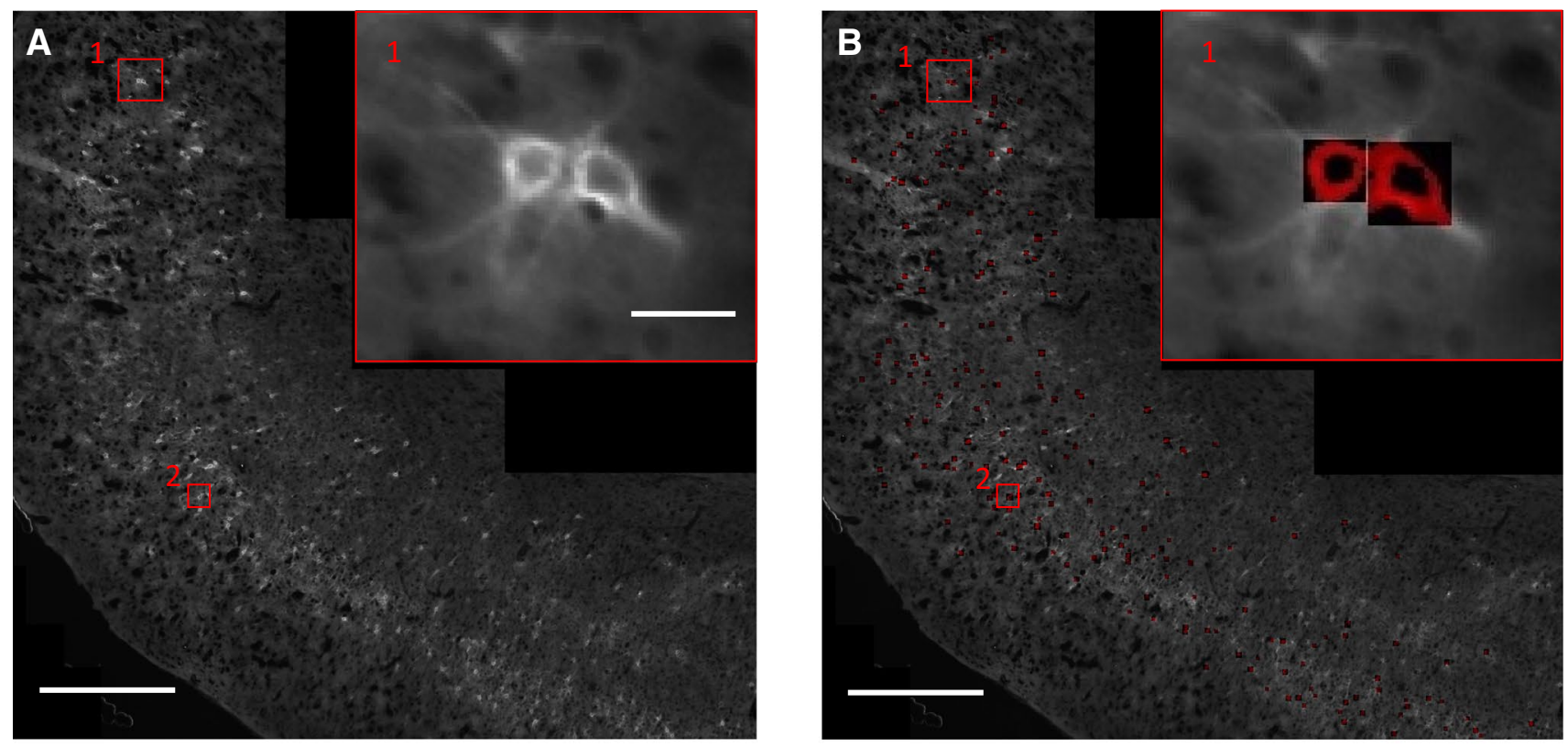

C

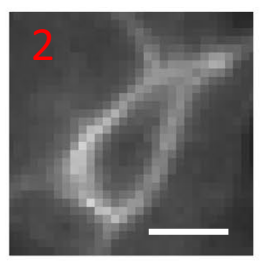

D
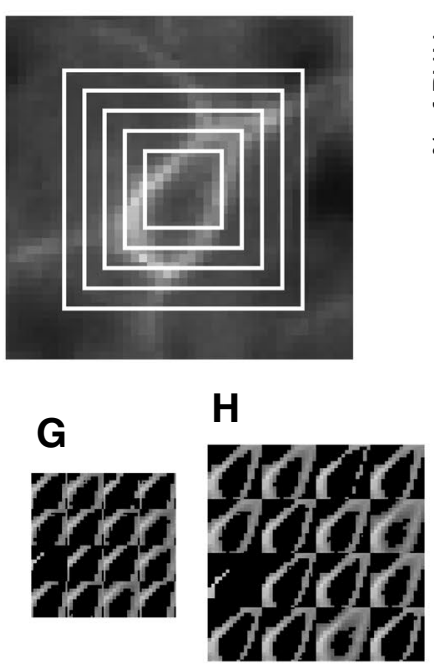

E

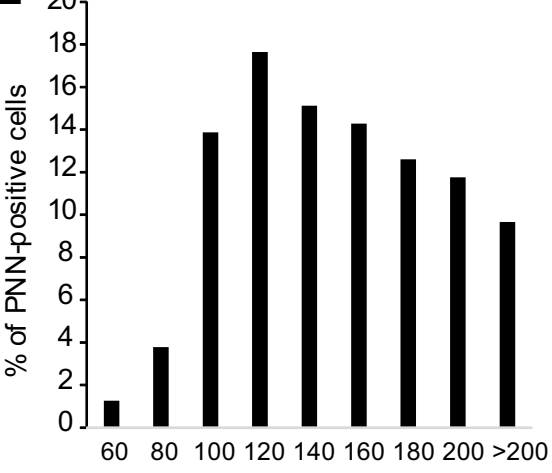

Fluorescence intensity, a.u.

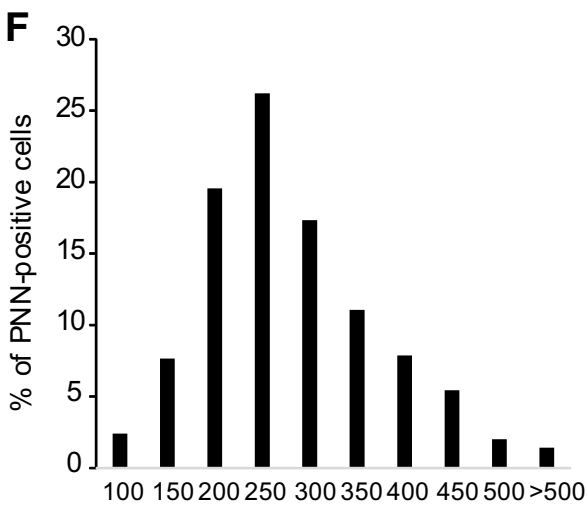

Area, $\mu \mathrm{m}^{2}$

$\mathbf{K}$

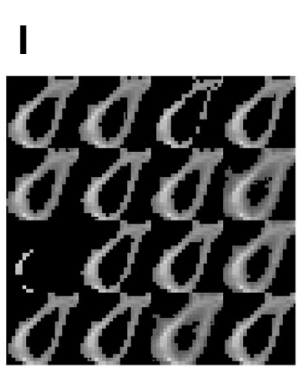

$\mathbf{J}$

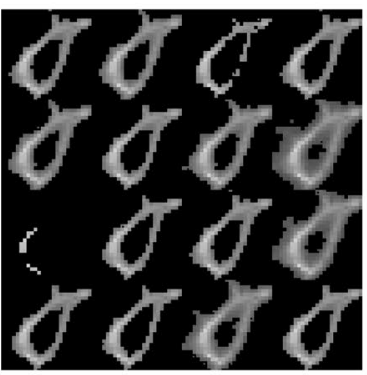

Fig. 1 PNN area and intensity quantification. a P28 mouse somatosensory cortex, coronal section stained with the PNN marker WFA. The red squares 1 and 2 indicate the areas shown at high magnification in the insert 1 and in (c). b Single cell masks mapped on the image in (a). Areas 1 and 2 correspond to those in (a). c A PNNbearing neuron center is marked manually. $\mathbf{d}, \mathbf{g}-\mathbf{k}$ Autothresholding is performed with 5 mask size variants—square edge size 10.2; 15.3;
20.4; 25.5; $30.6 \mathrm{~mm}$, and 16 autothresholding algorithms. e Single cell PNN mean intensity distribution histogram. Data are represented as mean for 4 autothresholding methods-Shanbhag, Intermodes, IsoData, Mean. f Single cell PNN area distribution histogram. Scale bar in (a) is $500 \mathrm{~mm}$, valid for (a, b), scale bar in the insert in (a) is 25 $\mathrm{mm}$, in (c) -10 mm, valid for $(\mathbf{c}, \mathbf{d})$, in $\mathbf{k}-25 \mathrm{~mm}$, valid for $(\mathbf{g}-\mathbf{k})$ 

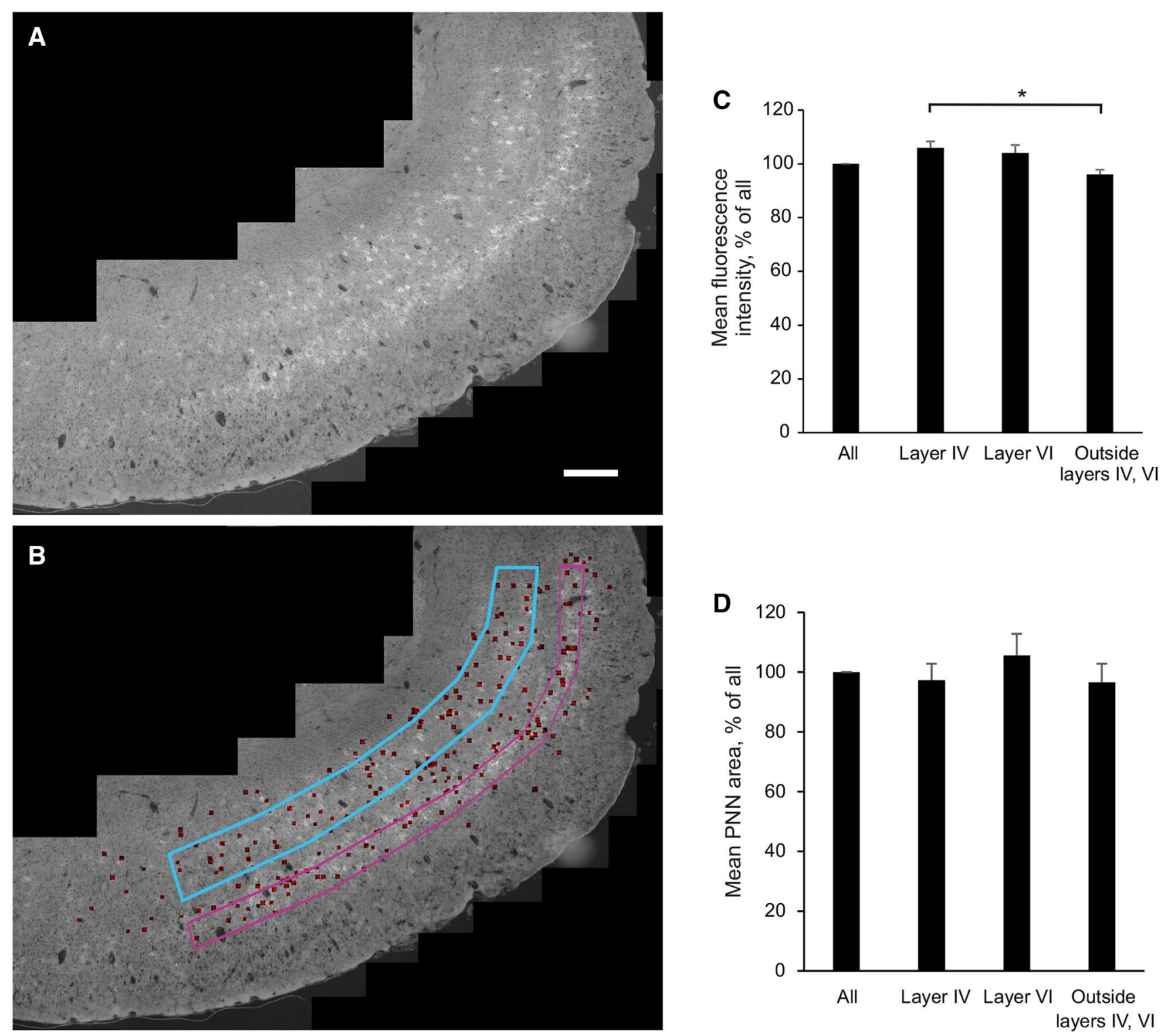

Fig. 2 PNN area and intensity in distinct regions of somatosensory cortex. a PNN-bearing cells are enriched in layers IV and VI of somatosensory cortex. $\mathbf{b}$ Single cell masks mapped on the image in $\mathbf{a}$. Cortical layers IV and VI are shown in purple and blue, respectively. c Mean values for single cell PNN chondroitin sulfate intensity-all cells, layers IV and VI, cells outside layers IV and VI. d Mean values for PNN area. Error bars represent standard error of mean (SEM), symbols * represent $P<0.05$. Scale bar in a is $300 \mu \mathrm{m}$, valid for $\mathbf{a , ~ b}$ and 0.001 , respectively. For the permutation test symbols \#, \#\# and \#\#\# represent $R<0.05,0.01$ and 0.001 , respectively.

\section{Results}

\section{PNN area and intensity quantification}

We used the intensity-based autothreshold procedure of the FIJI software to segment single cell PNN borders in histological sections of the adult mouse brain somatosensory cortex (Fig. 1a and Online Resource 3). For each single PNN-embedded neuron, the cell center was marked manually with the PointPicker tool (Fig. 1c). Next, a semi-automatic procedure was used to select appropriate segmentation area (i.e. cell size) for each single neuron. For each PNN-bearing neuron the appropriate cell size was chosen out of 5 variants, square size: $10.2 ; 15.3 ; 20.4 ; 25.5 ; 30.6$ $\mu \mathrm{m}(20,30,40,50,60$ pixels, respectively) (Fig. 1d, g-k). Autothresholding was performed with 16 different algorithms to ensure unbiased selection of the intensity threshold 


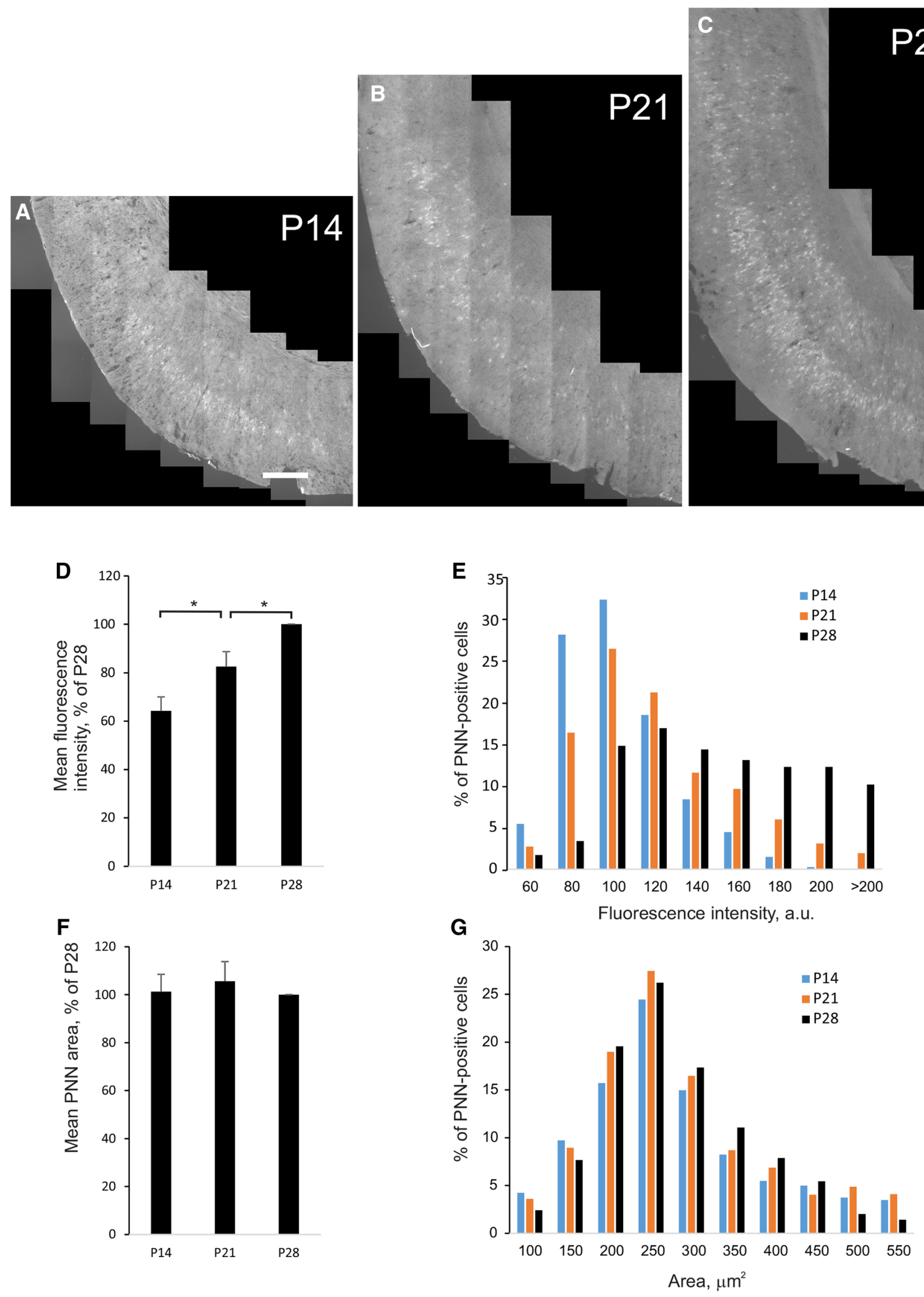


4 Fig. 3 Developmental changes in the somatosensory cortex PNN. a-c PNN in the mouse somatosensory cortex at P14, P21 and P28. d Mean intensity of the PNN chondroitin sulfate staining at P14, P21 and P28. e Single cell PNN intensity distribution histogram at P14, P21 and P28. f Mean area of the single cell PNN. g Single cell PNN area distribution histogram. Scale bar in $\mathbf{a}$ is $400 \mu \mathrm{m}$, valid for $\mathbf{a}-\mathbf{c}$

values. The set of autothresholding algorithms used in the present analysis produced 16 intensity values for the segmentation threshold resulting in a variety of the single cell PNN borders (Fig. 1g-k). The total number of 1079 PNNbearing neurons were analysed for cortical sections from 5 adult mice (Fig. 1b and Online Resource 4). Area and mean intensity were measured for the resulting masks of PNN surrounding individual neurons. We then tested convergence of the mean area values for single cell PNN based on 5 independent experiments and selected 4 algorithms (Shanbhag, Intermodes, IsoData, Mean) out of 16 that produced the most consistent quantification results. The single cell PNN intensity distribution histogram was extended on the right with intensity range of 120-200 arbitrary units being highly populated (Fig. 1e). The mean PNN intensity value was 152.8 a.u. $( \pm 37.2)$. The single cell PNN area histogram demonstrated Gaussian-type distribution (Fig. 1f) with the mean single cell PNN area value $289.3 \mu \mathrm{m}^{2}( \pm 100.1)$.

\section{PNN distribution in somatosensory cortex}

It was previously demonstrated that PNN-positive cells were enriched in the somatosensory cortical layers IV and VI (McRae et al. 2007). In the present study, we compared the PNN area and the chondroitin sulfate enrichment for the layers IV and VI (Fig. 2a, b and Online Resources 5, 6). The chondroitin sulfate staining intensity was higher for the layer IV as compared to the neurons located outside the layers IV and VI (Fig. 2c). No significant difference was observed for the PNN area between the studied regions (Fig. 2d).

\section{Developmental changes in the somatosensory cortex PNN}

We then used the same approach to quantify developmental dynamics of PNN maturation during the first postnatal month in juvenile mice. WFA staining for PNN was examined in the brain somatosensory cortex sections at the ages P14, P21 and P28 (Fig. 3a-c, Online Resources $7-12)$, the total number of 1822 neurons was analyzed from 9 mice, 3 independent experiments ( 202 neurons per mouse). The intensity of WFA staining raised gradually during the studied developmental period (Fig. 3d, e). Single cell PNN intensity distribution histograms demonstrate a profound developmental change of the chondroitin sulfate content distribution within the neuronal population. While the PNN intensity is characterized by a narrow peak of 50-100 arbitrary units at P14, the distribution profile changes remarkably at P21 and finally transforms into asymmetric distribution of the single cell intensities at P28 characterized by a broad extension of the histogram towards higher intensity values (Fig. 3e). By contrast, no significant changes were observed for the single cell PNN area (Fig. 3f, g). The mean area values for a single cell PNN were $286.7 \mu \mathrm{m}^{2}, 296.2 \mu \mathrm{m}^{2}$, and $282.5 \mu \mathrm{m}^{2}$ at P14, P21 and P28, respectively.

\section{PNN is differentially regulated following spinal cord injury}

The PNN CSPGs have been suggested to contribute to the mechanism of the regeneration failure after the spinal cord injury (Alilain et al. 2011; Massey et al. 2006). We therefore studied posttraumatic ipsilateral changes to the single cell PNN area and chondroitin sulfate staining intensity in the adult mouse spinal cord 9 weeks after cervical lateral hemisection in 7 injured mice, total number of neurons 3934. In the absence of spinal cord injury PNN area and intensity exhibit uniform distribution along the C3-C7 portion of the spinal cord as assessed for 4 control animals, total number of neurons 1980 (Fig. 4, Online Resource 13). By contrast, following the cervical lateral hemisection mean values for the PNN area were significantly lower within $200 \mu \mathrm{m}$ caudally from the injury site (Fig. 5a, Online Resource 14) as compared to the nearest proximal $200 \mu \mathrm{m}$ area rostrally from the injury site of the examined cervical spinal cord (Fig. 5d). Permutation test (Welch 1990) was used to verify significance for multiple data point comparison.

In the injured animals mean intensity values for single cell PNN were significantly higher at 1.4, 1.6 and $1.8 \mathrm{~mm}$ rostrally from the injury site in comparison to other portions of the examined cervical spinal cord (Fig. 5e).

\section{PNN density is increased caudally from the injury site}

We then quantified the density of PNN-bearing cells next to the injury site. We observed sustained increase of the PNN-bearing cell density in caudal direction (Fig. 6). This distribution was not observed in control uninjured animals (Online Resource 15). 


\section{Caudal}

Rostral

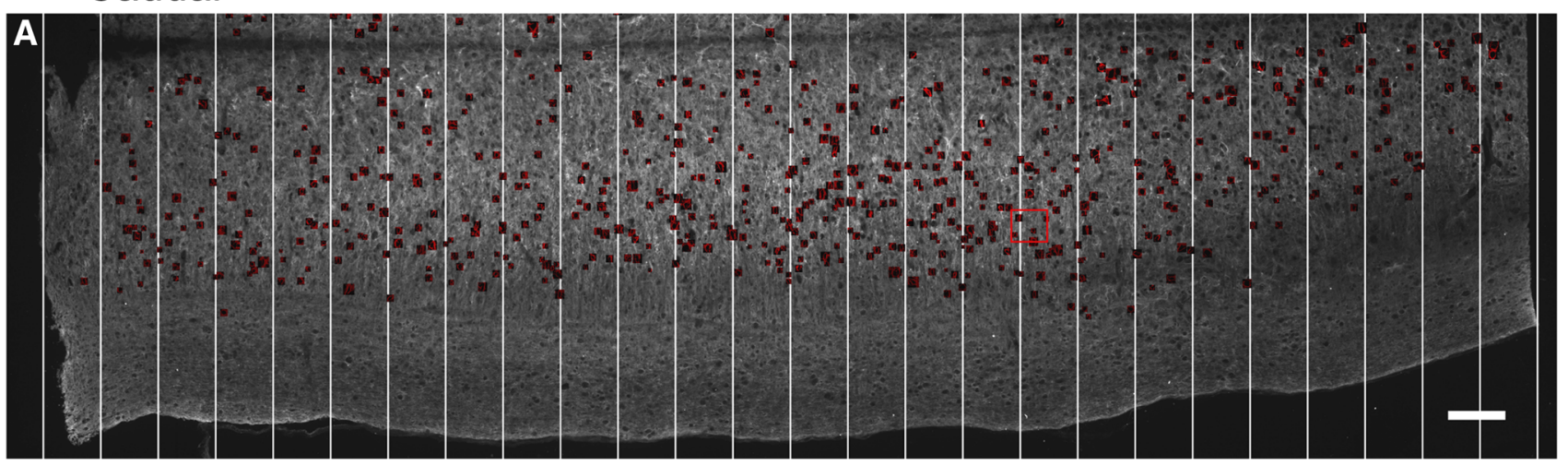

B

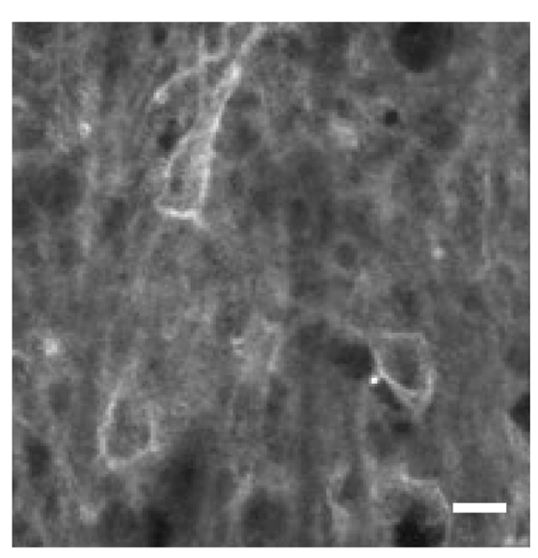

D

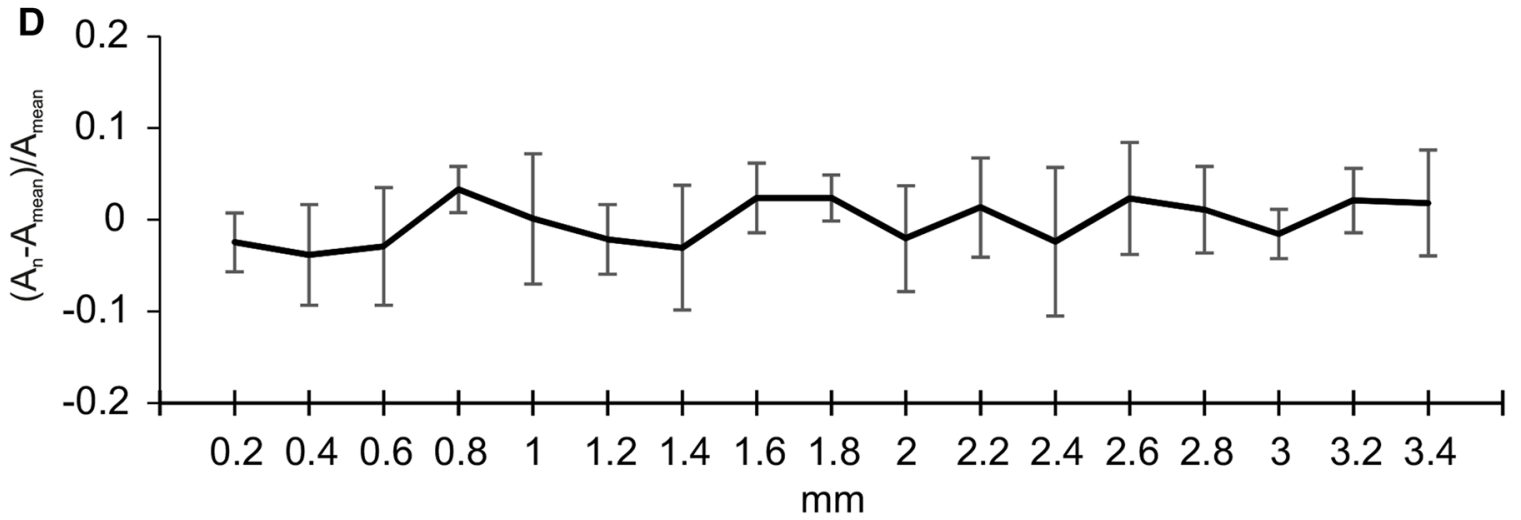


4Fig. 4 PNN in uninjured mouse cervical spinal cord. a Longitudinal horizontal section of the mouse cervical spinal cord at C3-C7 level, laminae 6, 7, stained with WFA. Single cell PNN masks are mapped on the fluorescent image. $\mathbf{b}$ High magnification of representative cells marked with a red rectangle in (a) c PNN masks for the cells shown in (b) d PNN area distribution along the cervical spinal cord. e PNN intensity distribution along the cervical spinal cord. PNN area and intensity are normalized as $\left(\mathrm{A}_{\mathrm{n}}-\mathrm{A}_{\text {mean }}\right) / \mathrm{A}_{\text {mean }}$ and $\left(\mathrm{I}_{\mathrm{n}}-\mathrm{I}_{\text {mean }}\right) / \mathrm{I}_{\text {mean }}$ in $\mathbf{d}$ and $\mathbf{e}$, respectively, where $\mathrm{A}_{\text {mean }}$ is average single cell PNN area value for each section, $\mathrm{I}_{\text {mean }}$ is average single cell PNN intensity value for each section. Data are averaged for 4 autothresholding algorithms: Default, Isodata, Otsu, Shanbhag, that give the best data convergence with each other. Data are shown for 4 independent experiments. The scale bar in $\mathbf{a}$ is $100 \mu \mathrm{m}$, in $\mathbf{b} 10 \mu \mathrm{m}$, valid for $\mathbf{b}, \mathbf{c}$

\section{The majority of PNN-positive neurons are parvalbumin-positive in the cervical intermediate grey}

The neurochemical type of PNN-positive neurons was previously studied in the rat spinal cord (Galtrey et al. 2008; Irvine and Kwok 2018; Vitellaro-Zuccarello et al. 2007). To address the neurochemical type of the PNN-positive neurons in the intermediate grey of the mouse cervical spinal cord we performed double staining with WFA and anti-parvalbumin IgG (Online Resources 16, 17). Total number of 771 neurons was analyzed from 3 mice. $83 \%$ of PNN-positive neurons were also parvalbumin-positive (Online Resource 18).

\section{Discussion}

Previous studies on PNN reported purely qualitative or limited quantitative assessment of the PNN microstructure during the CNS development and under posttraumatic conditions (McRae et al. 2007; Alilain et al. 2011; Massey et al. 2006). While developmental mRNA changes have been quantified for the PNN components including aggrecan, CRTL1, BRAL2, tenascin-R, HAS1 and 3 (Galtrey et al. 2008), the studies of the PNN size and intensity remained largely qualitative. Here we propose a semi-automatic approach for retrieval of quantitative parameters of PNN to be used for the studies of the development- and pathologyassociated changes in the CNS ECM.

The autothresholding-based approach used in the present study allows unbiased quantification of the major microstructural parameters of PNN (Fig. 1). The method presented here works on epifluorescent images suggesting that it can be widely applicable on regular microscopy equipment. Together with the semiautomatic procedure of the single cell PNN mask segmentation this suggests that the method can be highly efficient for the quantitative analysis of the PNN microstructure in large numbers (thousands) of individual cells. A limitation of the present method is that it is not fully automatic in terms of the selection of single cell positions and the size of segmentation area. In the present study we took advantage of the permutation test for quantitative comparison of multiple regions within the injured spinal cord based on the distance from the injury site (Figs. 5, 6). Our results suggest that the permutation test can serve as a highly valuable statistical tool especially for the comparison of large numbers of data points that is often the case for biomedical research.

Two other approaches were reported previously for the single cell PNN intensity quantification. One is based on random selection of 15 pixels within a WFA-positive perineuronal area (Foscarin et al. 2011). The method is applicable to limited cell numbers. Another method based on a region of interest construction around WFA-positive areas has been used for the analysis of large cell numbers in the model of cocaine addiction (Slaker et al. 2016, 2018). The method was not applied to the PNN area measurements.

PNN was reported in mouse somatosensory cortex as early as P10 (Nakamura et al. 2009) and is upregulated in various cortical regions between P14 and P30 (Ueno et al. 2017). In the rat spinal cord PNN mostly develop between P7 and P21 (Galtrey et al. 2008). Here we report gradual increase of the chondroitin sulfate GalNAc epitope enrichment in the mouse brain somatosensory cortex PNN during P14-P28 (Fig. 3d, e). The observed upregulation of chondroitin sulfate further supports the role of CSPG in the inhibition of synaptic plasticity during the closure of the critical period in mammalian brain (Miyata and Kitagawa 2015; Pizzorusso et al. 2002). Broadening of the single cell intensity distribution histogram between P14 and P28 reported in the present study (Fig. 3e) reflects changes of the chondroitin sulfate content within the PNN-bearing cell population and may be a characteristic feature of the PNN maturation during the closure of the critical period.

Yamada and co-authors previously compared chondroitin sulfate intensity in the PNN of parvalbumin-positive neurons in mouse hippocampus and dentate gyrus at P14, P60 and 12 months age (Yamada and Jinno 2013). In agreement with our data (Fig. 3) the authors report significant increase of the WFA staining intensity between P14 and P60 in the hippocampus and throughout the studied life period in dentate gyrus. Here we observed that the PNN area does not change significantly between P14 and P28 (Fig. 3f, g). This suggests that the extracellular volume occupied by PNN is set up early in postnatal development and preserves during the PNN maturation of somatosensory cortex. This finding may reflect the importance of the CSPG-enriched ECM for the ion homeostasis (Kwok et al. 2011), reactive oxygen species metabolism (Cabungcal et al. 2013) and for the tissue 

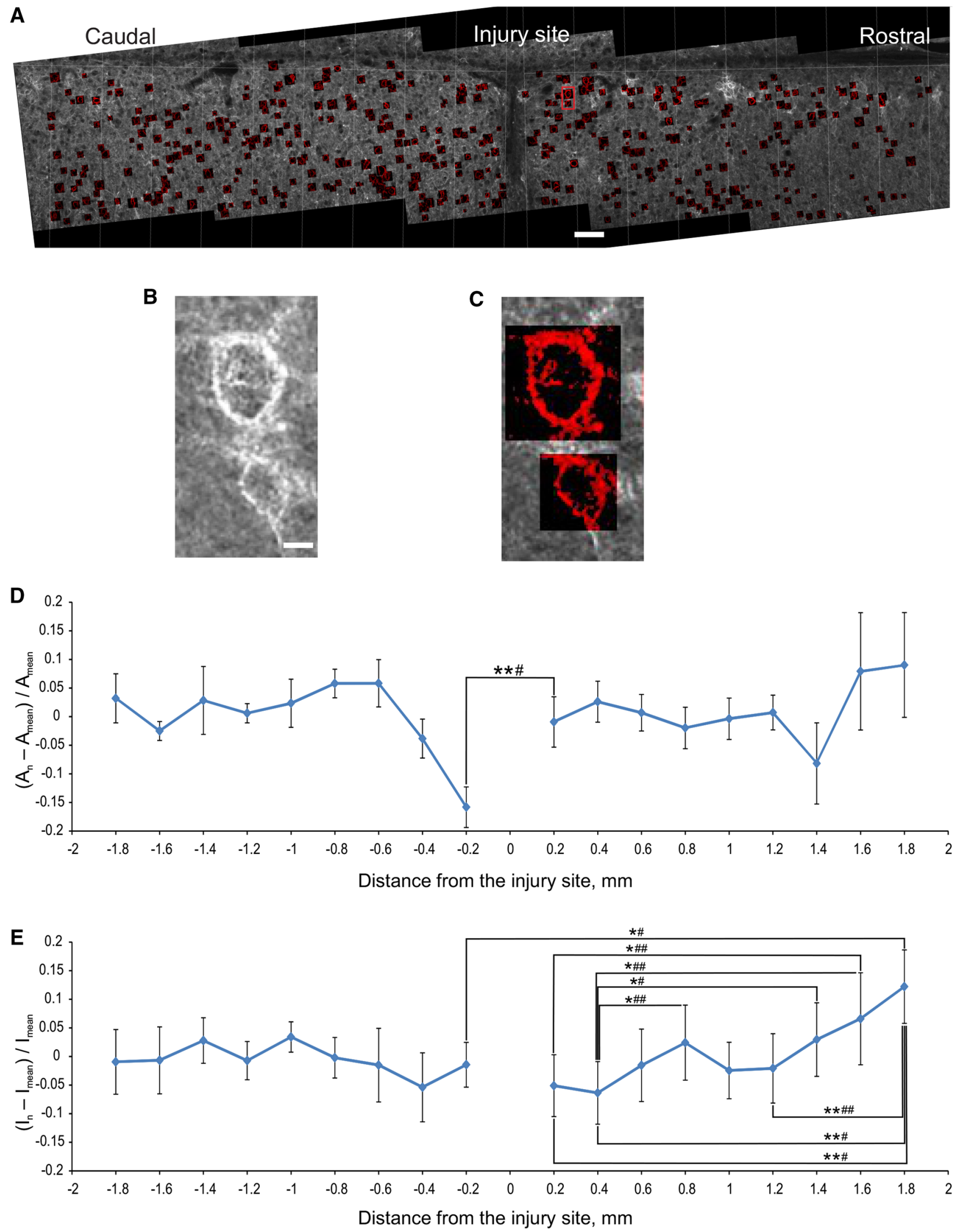
4Fig. $5 \mathrm{PNN}$ area and chondroitin sulfate intensity are differentially regulated in proximity to the injury site following spinal cord lateral hemisection. a Single cell PNN masks mapped on the longitudinal section of the cervical spinal cord subjected to lateral hemisection. b High magnification of representative cells marked with a red rectangle in a. c PNN masks for the cells shown in b. d Single cell PNN area distribution along the cervical spinal cord around the injury site at C5. e Single cell PNN chondroitin sulfate intensity distribution along the cervical spinal cord around the injury site at C5. Data are averaged for 4 autothresholding algorithms: Default, Isodata, Otsu, Shanbhag, that give the best data convergence with each other. Pairwise comparison is shown for data points with $P$ (black asterisk) and $R$ (magenta asterisk) values $<0.05$. The scale bar in $\mathbf{a}$ is $100 \mu \mathrm{m}$, in $\mathbf{b}$ $10 \mu \mathrm{m}$, valid for $(\mathbf{b}, \mathbf{c})$

distribution of extracellular signaling cues (Carulli et al. 2013; Kanato et al. 2009; Milev et al. 1998; Nandini et al. 2004) during the brain development and synaptic wiring.

The chondroitin sulfate moieties of PNN may contribute significantly to the inhibition of posttraumatic regeneration in the injured brain and spinal cord as CSPG digestion with chondroitinase $\mathrm{ABC}$ improves axonal regeneration in the injured spinal cord (Bradbury et al. 2002; García-Alías et al. 2009; Yick et al. 2003). The injury-induced upregulation of the PNN-associated CSPG was demonstrated in deafferented brainstem nuclei and around the spinal cord motor neurons far from the injury site (Alilain et al. 2011; Massey et al. 2005, 2006). In addition to that, axonal sprouting was restricted to the PNN- and CSPG-deficient regions in the posttraumatic brain cortex (Harris et al. 2010).

CSPG upregulation has been studied extensively in the posttraumatic glial scar as a major component of posttraumatic pathology causing inhibition of posttraumatic regeneration of the synaptic network (Cregg et al. 2014; Silver and Miller 2004). Upregulation of specific CSPGs including neurocan, brevican and versican along with changes in chondroitin sulfate sulfation patterns were reported in vicinity of the injury site (Jones et al. 2002, 2003; Buss et al. 2009). There is also a study demonstrating downregulation of aggrecan, neurocan and phosphacan around the injury site (Harris et al. 2009). Posttraumatic changes in the CSPG expression distantly from the injury site received less attention so far (Alilain et al. 2011; Massey et al. 2006). Those PNN changes were observed at 1-2 weeks after injury. The mechanism for the posttraumatic upregulation of the PNN CSPG may be associated with pro-inflammatory stimuli in deafferented nuclei (Alilain et al. 2011). In the present study we addressed long-lasting posttraumatic changes to the PNN size and intensity at 9 weeks after injury (Fig. 5). We demonstrate increase of the PNN chondroitin sulfate content at $1.6-1.8 \mathrm{~mm}$ rostrally from the injury site in laminae 6 and 7 grey matter around the central channel (Fig. 5e). In addition to that, the density of PNN-positive cells is significantly increased at $0.4-1.2 \mathrm{~mm}$ caudally from the injury site (Fig. 6). As revealed in the previous studies (Alilain et al. 2011; Massey et al. 2006) this upregulation of the PNN chondroitin sulfate may contribute to the posttraumatic inhibition of the synaptic network regeneration in the injured spinal cord. We further demonstrate decrease of the PNN area at $200 \mu \mathrm{m}$ caudally from the injury site (Fig. 5d), that is likely to reflect major posttraumatic changes in the nervous tissue adjacent to the injury site (Wanner et al. 2013; Cregg et al. 2014). The PNN area decrease reported in the present study may be caused by the superoxide production and oxidative stress in the injured CNS parenchima (Zhang et al. 2012). Indeed, chondroitin sulfate as well as another major PNN component hyaluronan can be degraded by reactive oxygen species (Rees et al. 2004).

In the present study we observed changes in the PNN area, CSPG enrichment and the density of PNN-bearing neurons within $1.8 \mathrm{~mm}$ rostrally and $1.2 \mathrm{~mm}$ caudally from the hemisection applied at $\mathrm{C} 5$. The cervical spinal cord portion (C3-C6) analyzed here corresponds to the phrenic motor nuclei location and the dorsal root entry zone for the phrenic afferents (Alilain et al. 2011; Nair et al. 2017). While phrenic motor neurons are located in the ventral horn, phrenic afferents project densely to the intermediate grey at C3-C6 (Nair et al. 2017), i.e. to the area analyzed in the present study (Figs. 4, 5, 6, Online Resources 13-18). Thus, the PNN-bearing neurons studied here may belong to the spinal cord synaptic circuitry of the respiration control. Our results suggest that the majority of those neurons are parvalbumin-positive interneurons (Online Resources 16-18). Respiratory disfunction is a severe consequence of the spinal cord injury (Schilero et al. 2018) underscoring the importance of posttraumatic neuronal plasticity in the studied area. The upregulation of the PNN chondroitin sulfate content at $1.6-1.8 \mathrm{~mm}$ rostrally from the injury site and the increase of the density of PNN-positive cells at $0.4-1.2 \mathrm{~mm}$ caudally from the injury site may serve as limiting factors for the posttraumatic regeneration of the phrenic motor control.

In summary, in the present study we reveal quantitative changes of the PNN structure during the CNS maturation and posttraumatic tissue reorganization. Our results suggest that the changes of the CSPG enrichment in PNN represent a common feature for the CNS early postnatal development and posttraumatic regeneration - the two states characterized by enhanced neuronal plasticity (Batty et al. 2017; Song and Dityatev 2018). The quantitative studies of the brain and spinal cord tissue microstructure performed in the present report could be further extended to address changes in the ECM associated with maturation of synaptic circuits and CNS injuries. 


\section{Caudal Injury site Rostral}

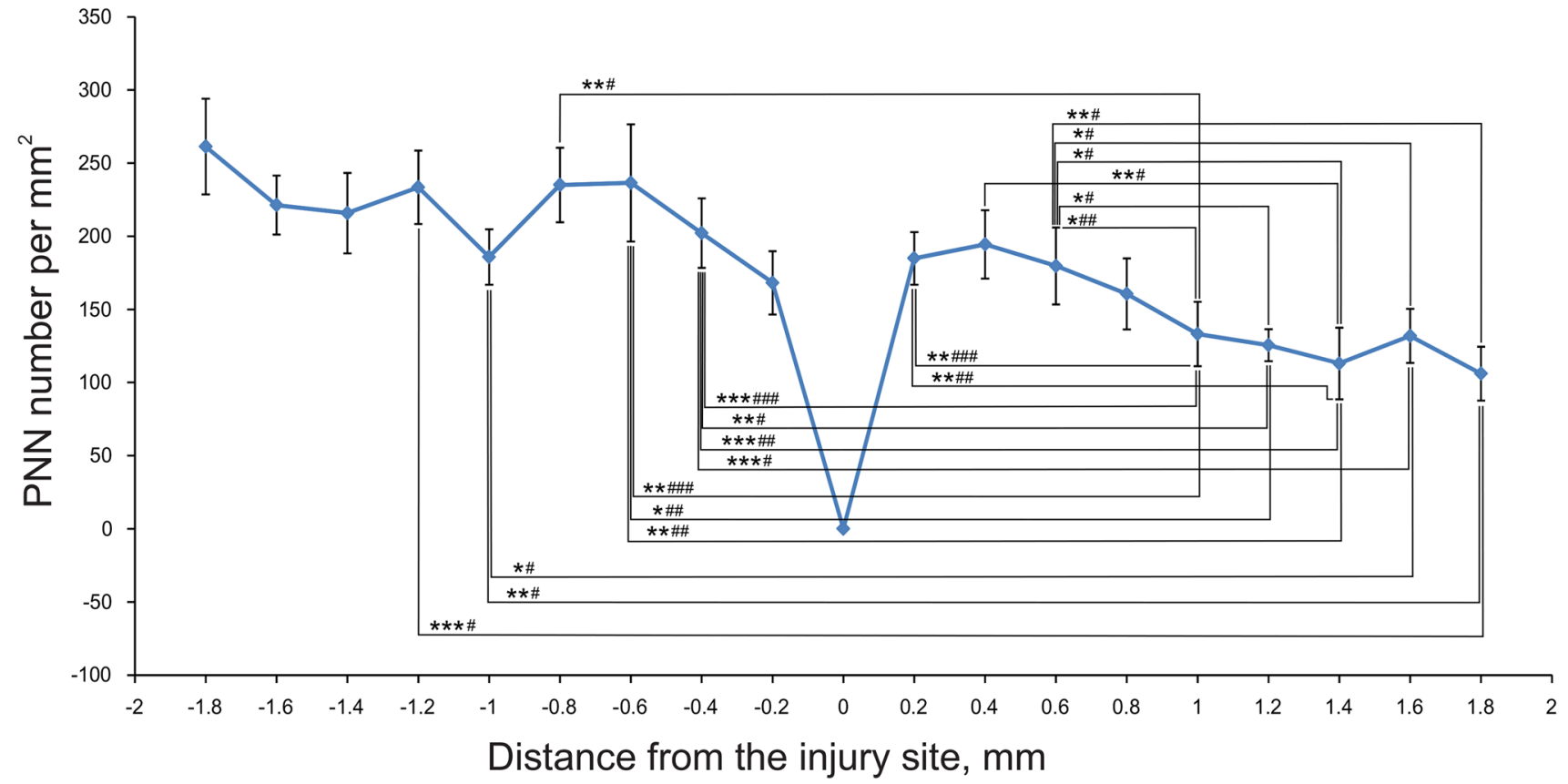

Fig. 6 PNN density is increased caudally from the injury site. The number of PNN-bearing neurons per mm ${ }^{2}$ was quantified at the distance $0.2-1.8 \mathrm{~mm}$ from the injury site. Pairwise comparison is shown for data points with $P$ (asterisk) and $R$ (hash mark) values $<0.05$

Acknowledgements Open access funding provided by University of Helsinki including Helsinki University Central Hospital. Arseniy Rascheskov and Nurislam Shaikhutdinov are acknowledged for assistance with histology and image analysis.

Funding MP was supported by the Academy of Finland Grant 1268543. The work was supported by Neuroscience Center, University of Helsinki. Sample preparation was supported by the Russian Science Foundation grant 14-15-00916. The work was performed according to the Russian Government Program of Competitive Growth of Kazan Federal University. NL was supported by a short-term grant from German Academic Exchange Service DAAD.

\section{Compliance with ethical standards}

Conflict of interest The authors declare that they have no conflict of interest.

Open Access This article is distributed under the terms of the Creative Commons Attribution 4.0 International License (http://creativeco mmons.org/licenses/by/4.0/), which permits unrestricted use, distribution, and reproduction in any medium, provided you give appropriate credit to the original author(s) and the source, provide a link to the Creative Commons license, and indicate if changes were made.

\section{References}

Alilain WJ, Horn KP, Hu H, Dick TE, Silver J (2011) Functional regeneration of respiratory pathways after spinal cord injury. Nature 475:196-200. https://doi.org/10.1038/nature10199

Arnst N, Kuznetsova S, Lipachev N, Shaikhutdinov N, Melnikova A, Mavlikeev M, Uvarov P, Baltina TV, Rauvala H, Osin YN, Kiyasov AP, Paveliev M (2016) Spatial patterns and cell surface clusters in perineuronal nets. Brain Res 1648:214-223. https://doi. org/10.1016/j.brainres.2016.07.020

Batty NJ, Fenrich KK, Fouad K (2017) The role of cAMP and its downstream targets in neurite growth in the adult nervous system. Neurosci Lett 652:56-63. https://doi.org/10.1016/j.neulet.2016.12.033

Bradbury EJ, Moon LD, Popat RJ, King VR, Bennett GS, Patel PN, Fawcett JW, McMahon SB (2002) Chondroitinase ABC promotes functional recovery after spinal cord injury. Nature 416:636-640. https://doi.org/10.1038/416636a

Brückner G, Kacza J, Grosche J (2004) Perineuronal nets characterized by vital labelling, confocal and electron microscopy in organotypic slice cultures of rat parietal cortex and hippocampus. J Mol Histol 35:115-122. https://doi.org/10.1023/B:HIJO.0000023374 .22298 .50

Burda JE, Bernstein AM, Sofroniew MV (2016) Astrocyte roles in traumatic brain injury. Exp Neurol 275:305-315. https://doi. org/10.1016/j.expneurol.2015.03.020

Buss A, Pech K, Kakulas BA, Martin D, Schoenen J, Noth J, Brook GA (2009) NG2 and phosphacan are present in the astroglial scar after human traumatic spinal cord injury. BMC Neurol 9:32. https ://doi.org/10.1186/1471-2377-9-32

Cabungcal JH, Steullet P, Morishita H, Kraftsik R, Cuenod M, Hensch TK, Do KQ (2013) Perineuronal nets protect fast-spiking interneurons against oxidative stress. Proc Natl Acad Sci USA 110:9130-9135. https://doi.org/10.1073/pnas.1300454110 
Carulli D, Pizzorusso T, Kwok JCF, Putignano E, Poli A, Forostyak S, Andrews MR, Deepa SS, Glant TT, Fawcett JW (2010) Animals lacking link protein have attenuated perineuronal nets and persistent plasticity. Brain 133:2331-2347. https://doi.org/10.1093/ brain/awq145

Carulli D, Foscarin S, Faralli A, Pajaj E, Rossi F (2013) Modulation of semaphorin3A in perineuronal nets during structural plasticity in the adult cerebellum. Mol Cell Neurosci 57:10-22. https://doi. org/10.1016/j.men.2013.08.003

Carulli D, Kwok JC, Pizzorusso T (2016) Perineuronal Nets and CNS plasticity and Repair. Neural Plast 2016:1-2. https://doi. org/10.1155/2016/4327082

Corvetti L, Rossi F (2005) Degradation of chondroitin sulfate proteoglycans induces sprouting of intact Purkinje axons in the cerebellum of the adult rat. J Neurosci 25:7150-7158. https://doi. org/10.1523/JNEUROSCI.0683-05.2005

Cregg JM, DePaul MA, Filous AR, Lang BT, Tran A, Silver J (2014) Functional regeneration beyond the glial scar. Exp Neurol 253:197-207. https://doi.org/10.1016/j.expneurol.2013.12.024

Dityatev A, Rusakov DA (2011) Molecular signals of plasticity at the tetrapartite synapse. Curr Opin Neurobiol 2:353-359. https://doi. org/10.1016/j.conb.2010.12.006

Fawcett JW (2015) The extracellular matrix in plasticity and regeneration after CNS injury and neurodegenerative disease. Brain Res 218:213-226. https://doi.org/10.1016/bs.pbr.2015.02.001

Foscarin S, Ponchione D, Pajaj E, Leto K, Gawlak M, Wilczynski GM, Rossi F, Carulli D (2011) Experience-dependent plasticity and modulation of growth regulatory molecules at central synapses. PLoS ONE 6:e16666. https://doi.org/10.1371/journal.pone.00166 66

Galtrey CM, Asher RA, Nothias F, Fawcett JW (2007) Promoting plasticity in the spinal cord with chondroitinase improves functional recovery after peripheral nerve repair. Brain 130:926-939. https ://doi.org/10.1093/brain/awl372

Galtrey CM, Kwok JC, Carulli D, Rhodes KE, Fawcett JW (2008) Distribution and synthesis of extracellular matrix proteoglycans, hyaluronan, link proteins and tenascin- $\mathrm{R}$ in the rat spinal cord. Eur J Neurosci 27:1373-1390. https://doi.org/10.111 1/j.1460-9568.2008.06108.x

García-Alías G, Barkhuysen S, Buckle M, Fawcett JW (2009) Chondroitinase $\mathrm{ABC}$ treatment opens a window of opportunity for task-specific rehabilitation. Nat Neurosci 9:1145-1151. https:// doi.org/10.1038/nn.2377

Giamanco KA, Morawski M, Matthews RT (2010) Perineuronal net formation and structure in aggrecan knockout mice. Neuroscience 170:1314-1327. https://doi.org/10.1016/j.neuroscien ce.2010.08.032

Harris NG, Carmichael ST, Hovda DA, Sutton RL (2009) Traumatic brain injury results in disparate regions of chondroitin sulfate proteoglycan expression that are temporally limited. J Neurosci 87:2937-2950. https://doi.org/10.1002/jnr.22115

Harris NG, Mironova YA, Hovda DA, Sutton RL (2010) Pericontusion axon sprouting is spatially and temporally consistent with a growth-permissive environment after traumatic brain injury. $\mathrm{J}$ Neuropathol Exp Neurol 69:139-154. https://doi.org/10.1097/ NEN.0b013e3181cb5bee

Härtig W, Brauer K, Brückner G (1992) Wisteria floribunda agglutinin-labelled nets surround parvalbumin-containing neurons. Neuroreport 3:869-872. https://doi.org/10.1097/00001756-19921 0000-00012

Irvine SF, Kwok JCF (2018) Perineuronal Nets in Spinal Motoneurones: Chondroitin Sulphate Proteoglycan around Alpha Motoneurones. Int J Mol Sci 19:1172. https://doi.org/10.3390/ijms1 9041172

Jones LL, Yamaguchi Y, Stallcup WB, Tuszynski MH (2002) NG2 Is a major chondroitin sulfate proteoglycan produced after spinal cord injury and is expressed by macrophages and oligodendrocyte progenitors. J Neurosci 22:2792-2803

Jones LL, Margolis RU, Tuszynski MH (2003) The chondroitin sulfate proteoglycans neurocan, brevican, phosphacan, and versican are differentially regulated following spinal cord injury. Exp Neurol 182:399-411. https://doi.org/10.1016/S0014-4886(03)00087-6

Kanato Y, Ono S, Kitajima K, Sato C (2009) Complex formation of a brain-derived neurotrophic factor and glycosaminoglycans. Biosci Biotechnol Biochem 73:2735-2741

Korotchenko S, Zanacchi FC, Diaspro A, Dityatev A (2014) Zooming in on the perisynaptic extracellular matrix. Nanoscale Imaging Synapses 84:187-203. https://doi.org/10.1007/978-1-4614-9179-8_10

Kwok JCF, Carulli D, Fawcett JW (2010) In vitro modeling of perineuronal nets: Hyaluronan synthase and link protein are necessary for their formation and integrity. J Neurochem 114:1447-1459. https ://doi.org/10.1111/j.1471-4159.2010.06878.x

Kwok JC, Dick G, Wang D, Fawcett JW (2011) Extracellular matrix and perineuronal nets in CNS repair. Dev Neurobiol 71:10731089. https://doi.org/10.1002/dneu.20974

Lander C, Zhang H, Hockfield S (1998) Neurons produce a neuronal cell surface-associated chondroitin sulfate proteoglycan. J Neurosci 18:174-183. https://doi.org/10.1523/JNEUROSCI.18-0100174.1998

Massey JM, Matthews RT, Viapiano MS, Kalyfa A, Wagoner M, Decker JA, Cooper NGF, Onifer SM (2005) Chondroitin sulfate proteoglycan expression within the adult rat brainstem dorsal column nuclei following cervical spinal cord injury. Soc Neurosci $31: 836$

Massey JM, Hubscher CH, Wagoner MR, Decker JA, Amps J, Silver J, Onifer SM (2006) Chondroitinase ABC digestion of the perineuronal net promotes functional collateral sprouting in the cuneate nucleus after cervical spinal cord injury. J Neurosci 26:44064414. https://doi.org/10.1523/JNEUROSCI.5467-05.2006

McRae PA, Rocco MM, Kelly G, Brumberg JC, Matthews RT (2007) Sensory deprivation alters aggrecan and perineuronal net expression in the mouse barrel cortex. J Neurosci 27:5405-5413. https ://doi.org/10.1523/JNEUROSCI.5425-06.2007

Milev P, Chiba A, Häring M, Rauvala H, Schachner M, Ranscht B, Margolis RK, Margolis RU (1998) High affinity binding and overlapping localization of neurocan and phosphacan/proteintyrosine phosphatase-zeta/beta with tenascin-R, amphoterin, and the heparin-binding growth-associated molecule. J Biol Chem 273:6998-7005. https://doi.org/10.1074/jbc.273.12.6998

Miyata S, Kitagawa H (2015) Mechanisms for modulation of neural plasticity and axon regeneration by chondroitin sulphate. J Biochem 157:13-22. https://doi.org/10.1093/jb/mvu067

Nair J, Bezdudnaya T, Zholudeva LV, Detloff MR, Reier PJ, Lane MA, Fuller DD (2017) Histological identification of phrenic afferent projections to the spinal cord. Respir Physiol Neurobiol 236:5768. https://doi.org/10.1016/j.resp.2016.11.006

Nakamura M, Nakano K, Morita S, Nakashima T, Oohira A, Miyata S (2009) Expression of chondroitin sulfate proteoglycans in barrel field of mouse and rat somatosensory cortex. Brain Res 1252:117129. https://doi.org/10.1016/j.brainres.2008.11.022

Nandini CD, Mikami T, Ohta M, Itoh N, Akiyama-Nambu F, Sugahara K (2004) Structural and functional characterization of oversulfated chondroitin sulfate/dermatan sulfate hybrid chains from the notochord of hagfish. Neuritogenic and binding activities for growth factors and neurotrophic factors. J Biol Chem 279:50799-50809

Pizzorusso T, Medini P, Berardi N, Chierzi S, Fawcett JW, Maffei L (2002) Reactivation of ocular dominance plasticity in the adult visual cortex. Science 298:1248-1251. https://doi.org/10.1126/ science. 1072699

Rees MD, Hawkins CL, Davies MJ (2004) Hypochlorite and superoxide radicals can act synergistically to induce fragmentation of 
hyaluronan and chondroitin sulphates. Biochem J 381:175-184. https://doi.org/10.1042/BJ20040148

Schilero GJ, Bauman WA, Radulovic M (2018) Traumatic spinal cord injury: pulmonary physiologic principles and management. Clin Chest Med 39:411-425. https://doi.org/10.1016/j. ccm.2018.02.002

Schindelin J, Arganda-Carreras I, Frise E, Kaynig V, Longair M, Pietzsch T, Preibisch S, Rueden C, Saalfeld S, Schmid B, Tinevez JY, White DJ, Hartenstein V, Eliceiri K, Tomancak P, Cardona A (2012) Fiji: an open-source platform for biological-image analysis. Nat Methods 9:676-682. https://doi.org/10.1038/nmeth.2019

Shen HH (2018) Core concept: perineuronal nets gain prominence for their role in learning, memory, and plasticity. Proc Natl Acad Sci USA 115:9813-9815. https://doi.org/10.1073/pnas.1815273115

Silver J, Miller JH (2004) Regeneration beyond the glial scar. Nat Rev Neurosci 5:146-156. https://doi.org/10.1038/nrn1326

Slaker ML, Harkness JH, Sorg BA (2016) A standardized and automated method of perineuronal net analysis using Wisteria floribunda agglutinin staining intensity. IBRO Rep 1:54-60. https:// doi.org/10.1016/j.ibror.2016.10.001

Slaker ML, Jorgensen ET, Hegarty DM, Liu X, Kong Y, Zhang F, Linhardt RJ, Brown TE, Aicher SA, Sorg BA (2018) Cocaine exposure modulates perineuronal nets and synaptic excitability of fast-spiking interneurons in the medial prefrontal cortex. eNeuro. https://doi.org/10.1523/ENEURO.0221-18.2018

Song I, Dityatev A (2018) Crosstalk between glia, extracellular matrix and neurons. Brain Res Bull 136:101-108. https://doi. org/10.1016/j.brainresbull.2017.03.003

Ueno H, Suemitsu S, Okamoto M, Matsumoto Y, Ishihara T (2017) Parvalbumin neurons and perineuronal nets in the mouse prefrontal cortex. Neuroscience 343:115-127. https://doi.org/10.1016/j. neuroscience.2016.11.035
Vitellaro-Zuccarello L, Bosisio P, Mazzetti S, Monti C, De Biasi S (2007) Differential expression of several molecules of the extracellular matrix in functionally and developmentally distinct regions of rat spinal cord. Cell Tissue Res 327:433-447

Wanner IB, Anderson MA, Song B, Levine J, Fernandez A, GrayThompson Z, Ao Y, Sofroniew MV (2013) Glial scar borders are formed by newly proliferated, elongated astrocytes that interact to corral inflammatory and fibrotic cells via STAT3-dependent mechanisms after spinal cord injury. J Neurosci 33:12870-12886. https://doi.org/10.1523/JNEUROSCI.2121-13.2013

Welch WJ (1990) Construction of permutation tests. J Am Stat Assoc 85:693-698. https://doi.org/10.1080/01621459.1990.10474929

Yamada J, Jinno S (2013) Spatio-temporal differences in perineuronal net expression in the mouse hippocampus, with reference to parvalbumin. Neuroscience 253:368-379. https://doi.org/10.1016/j. neuroscience.2013.08.061

Yick LW, Cheung PT, So KF, Wu W (2003) Axonal regeneration of Clarke's neurons beyond the spinal cord injury scar after treatment with chondroitinase ABC. Exp Neurol 182:160-168. https://doi. org/10.1016/S0014-4886(02)00052-3

Zhang QG et al (2012) Critical role of NADPH oxidase in neuronal oxidative damage and microglia activation following traumatic brain injury. PLoS ONE 7:e34504. https://doi.org/10.1371/journ al.pone.0034504

Publisher's Note Springer Nature remains neutral with regard to jurisdictional claims in published maps and institutional affiliations. 